\title{
État actuel de l'Appareillage électrique d'interruption pour hautes tensions alternatives.
}

\author{
Par G. GERIN, des Elablissements Merlin etGerin, à Grenoble.
}

\section{Historique}

Au cours des premiers temps du développement des applications électriques on se contentait de transporter seulement sur de courtes distances une faible quantité d'énergie.

Cette dernière suffisait alors pour les besoins du moment. Mais par suite de la rapide croissance du développement des applications de l'électricité, les réseaux de distribution ont pris des proportions énormes. On transporte aujourd'hui à plusicurs centaines de kilomètres des puissances pouvant atteindre plusicurs centaines de milliers de K.V.A.

Tant qu'on opérait sur de faibles puissances, la mise en circuit et hors circuit des lignes ou appareils d'utilisation se faisait de la manière la plus simple et personne ne se doutait que ceci était le sujet d'un problème destiné à devenir si important plus tard et qui n'est pas encore complètement résolu.

Ce premier type d'interrupteur est celui appelé communément aujourd'hui "sectionneur ". A l'origine, il différail très peu de l'interrupteur à couteau B. T. et e'est d'ailleurs de la forme de ce dernier que les constructeurs s'inspirèrent.

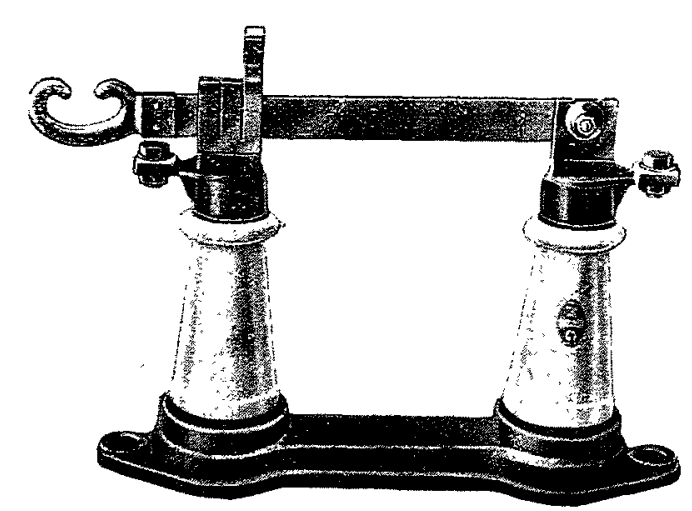

Sectionneur unipolaire sur isolateurs lisses 15.000 volts 250 ampères.

Ceci revient à dire qu'on ne faisait pas de différence entre l'appareil de manœuvre pour basse tension et haute tension, mais avec des puissances croissantes, quoique encore bien inféricures à celles rencontrées de nos jours, on s'aperçut que le sectionneur était un appareil peu sûr pour couper en charge, parce que chaque interruption faite dans ces conditions donnait lieu à un arc, qui prenait dans l'air un développement considérable, détériorant l'appareil et donnant lieu parfois à des accidents de personne.

L'attention des constructeurs fut alors appelée sur cet arc. Comme on ne possédait pas encore de moyens très efficaces pour parer à ses effets, on se contenta de chercher à le souffler.

On fit alors des appareils à soufflage magnétique encore en faveur pour les distributions à courant continu, mais qui furent ensuite rapidement abandonnés pour céder la place à l'interrupteur à cornes dans lequel le soufflage est obtenu d'une manière en quelque sorte automatique, en utilisant la vitesse du courant d'air ascendant provoqué par l'échauffement de l'air ambiant, et sans l'intervention de bobines destinées à créer un champ magnélique.

Ce dispositif fut inspiré par le parafoudre à cornes, alors déjà connu, et subsiste encore de nos jour's pour les faibles puissances, sous la désignation "d'interrup leur aérien ".

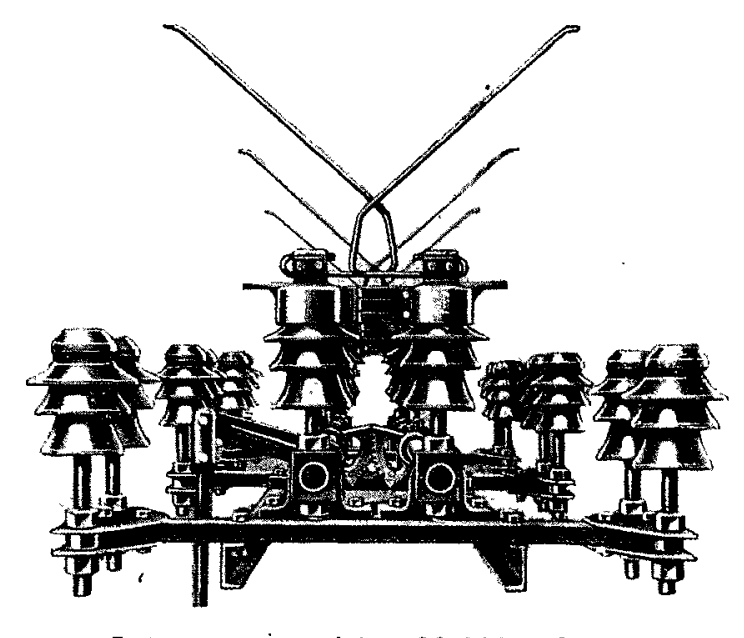

Interrupteur aćrien 30.000 volts.

Lorsque l'énergie transportée augmenta encore, on éleva avec elle la tension de distribulion. Il fut impossible de continuer à admettre un appareil de manceuvre domnant lieu à un are dans l'air, car celui-ci prenait alors un développement par trop considérable. On eut l'idée d'effectuer la rupture dans l'huile de pétrole déjà utilisée pour l'isolement des transformateurs.

Ce fut là un progrès décisif dans la construction de l'appareillage d'interruption.

C'est depuis ce temps qu'il devint possible de rompre des puissances notables à des tensions croissant de jour en jour.

Aujourd'hui, l'exploitant a à sa disposition des appareils dont la capacité d'interruption cerlaine dépasse la limite des plus fortes puissances transportées.

\section{EFFoRTS RÉALISÉS}

Ce n'est pas tout de suite cependant que l'on parvint à construire des appareils aussi sûrs.

Les constructeurs tâtonnèrent longtemps et ce fut le règne d'un empirisne présidant scul à la détermination des appareils.

La construction actuelle est le fruit d'une longue pralique dont toutes les expériences ont été disséquées à la lumière des données scientifiques et aussi parfois de longues el palientes recherches.

En particulier, ce n'est que lentement qu'on s'expliqua les phénomènes de rupture à travers l'huile.

On crut tout d'abord que la rupture érait effectuée au seill du liquide. Ce fut seulement le jour où l'on eul pénélré le processus physique du phénomène de l'arc électrique, que l'on s'aperçut que ceci était incompatible avec les condilions même d'existence de ce phénomène.

On conçut alors l'hypothèse d'une vaporisation spontanée d'une certaine quantité d'huile formant dans son sein une masse gazeuse à l'in térieur de laquelle l'are peut se développer librement.

On chercha ensuite quel était l'effet sur l'arc de rupture des constantes actuclles du réseau ? On différencia la coupure ell charge inductive de celle en charge ohmique ; on se préoccupa 
aussi des surtensions d'extra-courant de ruplure, et on éludia l'ufluence soit d'une résistance, soit d'une réaclance insérée en série dans les circuits au moment de la rupture.

De celle dernière élude subsiste aujourd'hui l'appareil à résistillec de choc.

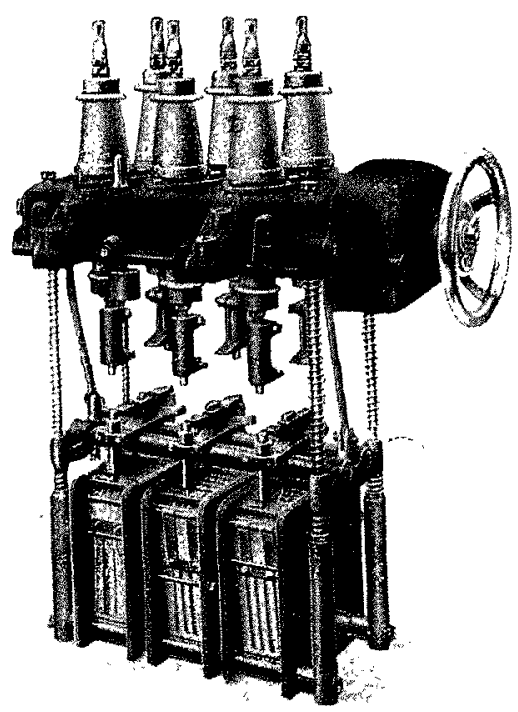

Disjoncteur dans l'huile 10.000 volts, 250 amp. muni de résistance de choc.

On sentit le besoin d'effecluer des expériences sérieuses de ma nière à contrôler rigoureusement l'exactitude dés hypothèses énises, de délimiter leur champ d'application, et enfin, d'en déduire des règles précises permettant la prédétermination exacte d'un appareil opérant avec toute la sécurité désirable.

Nous ne passerons pas en revue les diverses tentatives et ne décrirons pas tout au long les expériences effectuées : nous nous bornerons à ciler les mémoires de Meriam (Transactions of the A. I. E. E. Vol. XXX), de Ryndall et de Collis (Transactions of the A. I. E. E. Vol. XXXIV), en Amérique, et celui de Bruno Bauer (Bulletin de l'A. S. E. 1917, fascicules 9 et 10), en Suisse.

A l'heure actuelle, le mécanisme de la rupture dans l'huile est expliqué comme suit :

Au moment de la séparation des contacts, la résistance de contact devient telle que l'anode est vaporisée, la cathode est échauffée, et que par conséquent, la mince pellicule d'huile qui s'introduit entre les électrodes est également vaporisée ; à ce moment l'are s'élablit. Aufur et à mesure que les contacts sont éloignés, il se développe dans unc enceinte libre d'huile dont le volume augmente d'ailleurs par suite de la haute lempérature de l'are et croît ainsi jusqu'à l'extinction.

L'arc passe donc par toutes les phases successives. Il agil sur le circuil comme une résistance variable, pour une cerlaine valeur de celte résistance, l'arc est slable, puis il devient de plus en plus instable et s'éteint définitivement.

Nous ne savons pas quelle est la loi de variation de la résistance introduite en sćrie dans le circuil par l'are, mais nous savons qu'elle se différencic de celle d'un are stationnaire en ee sens qu'elle est fonction de la distance des électrodes. Le mème phénomène a lieu d'ailleurs avec les apparcils à cornes.

Nous ne savons pas non plus tenir compte de liomisation due à l'arc el aux électrodes incandescents. Néanmoins, il y a lout licu de croire que celle-ci n'a pas grande influence sur le développement de l'are alternatif à basse frécuence. En effet, la durée de la période d'extinction au passage du courant par zéro, est suffisante pour que la recombinaison des ions, antéricurcment formés soit pratiquement complète, et que ceux-ci $n$ arent pas d'influence sur la nouvelle période de rallumage de l'arc.

En outre, tous les expérimentaleurs sont d'accord pour admettre que la coupure dans l'huile, par suite de la formation d'une masse gazeuse et de la pression qui résulte de sa température, donne lieu, au moment de la détente du gaz, à un travail mécanique appelé "Travail de rupture ». Le travail de rupture met en jeu une certaine quantité d'énergie dite énergie de rupture, empruntée à l'énergie électrique du système interrompu. C'est le mémoire de Bruno Bauer qui donne le plus de précision à ce sujet. Il permet de calculer le travail de rupture et de dimensionner l'appareil en conséquence.

On définit ainsi la capacité de rupture d'un interrupteur, c'est-à-dire la plus grande puissance interruptible en toute sécurité ; c'est le produit du plus fort courant de court-circuit compatible avec les conditions de l'installation par la tension normale de service supposée maintenue. Cette dernière supposition est évidemment fausse et fournit des résultats erronés par excès. Ceci explique que la capacité de rupture ait le caractère d'une valeur limite. Toutefois, il est nécessaire de se mettre en garde contre la supposition actuelle qu'en court-circuit la tension tombe à zéro. Il ne faut pas oublier que les générateurs modernes peuvent avoir une réactance interne atteignant 15 à $35 \%$.

Enfin, on n'est pas assez sévère en général dans l'évaluation du rapport du courant de court-circuit permanent au courant normal de l'alternateur.

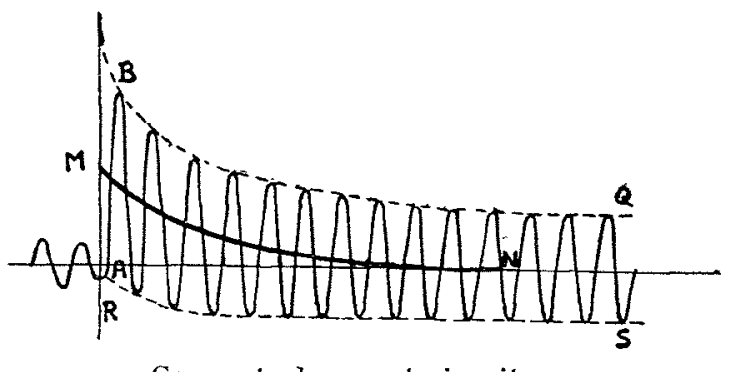

Courant de court-circuit.

On suppose ordinairement que ce rapport est égal à 3 , mais nous rappelons à ce sujet qu'il existe d'excellentes études de la question, l'une des plus suggestives se trouve dans un mémoire de O. R. Doherty et O. E. Shirley (Transactions of the A. I. E. E. Vol. XXXVII) qui donnent sous forme de tableau les résultals d'essais de courts-circuits effectués sur une trentaine de machines de caractéristiques diverses du typo à pôles saillants et pour lesquelles ils trouvent des valeurs du rapport précédent variant entre 3,5 et 4,5 mais atteignant parfois 6 .

Il y a lieu de remarquer, en outre, que le courant de courtcircuit transitoire ou courant d'établissement du court-circuit peut alteindre des valeurs beaucoup plus considérables (jusqu'à 20 ou 30 fois le courant normal) (Diamant: Suden short-circuit Phenomene and transient armature reactance of alternators. Transactions of the A. I. E. E., vol. XXXVII); mais, il est inutile des'en inquiéter au moment de la ruplure, car la durée de la période d'établissement du courant de court-circuit est telle que l'inertic des pièces mécaniques produisant celle rupture permel de la dépasser largement.

Le plus important progrès de ces dernières années consiste précisément à savoir détcrminer le type d'interrupteur convenable pour une installation, ou des condilions, domnées. En effet, cn aucun cas, on ne doit admettre d'appareil dont la capacité de rupture soit inférieure à la puissance maximum interruptible. Il appartient à l'exploitant de limiter par des dispositifs particuliers la valeur des courants des courts-circuits maxima pour no pas imposer aux appareils de son réseau des dimensions 
incompatibles avec unc distribution économique. Les appartils couramment employés à cet effet sont les bobines de réactance. Disons, en passant, que c'est là un puissant moven de réduction du courant de court-circuit à la fois en régime transitoirc et permanent, et dont l'emploi judicieux permet de prévenir les accidenis aus au premier régime de ce courant.

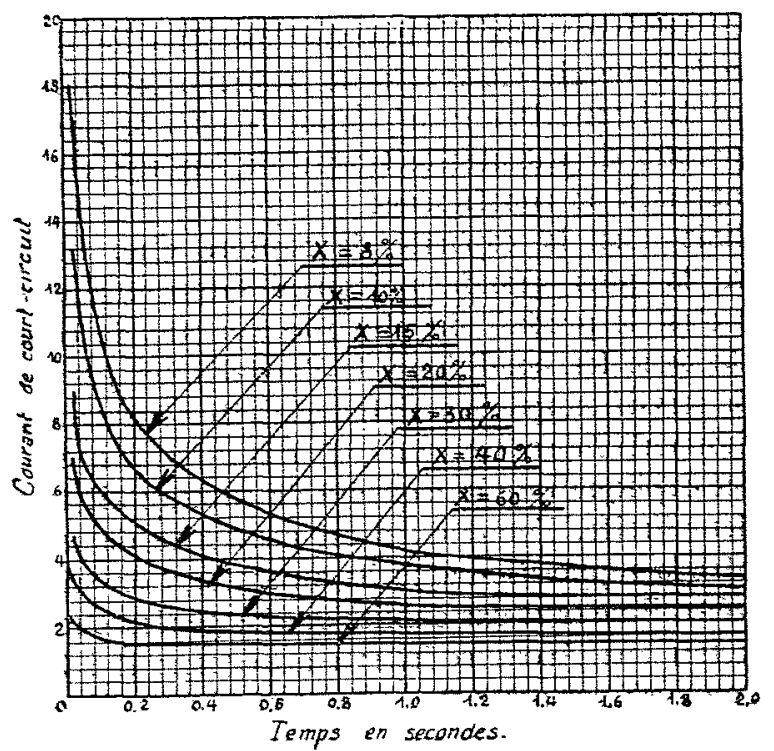

Courbes pour les déterminations de courant de court-circuit en fonction du temps, éc de la réactance sur le réseau.

En outre, l'emploi de relais temporisés permet l'établisscment du courant permanent de court-circuit el par conséquent la diminution de la puissance interruptible au moment de la coupure. Enfin, c'est grâce au relais sélectionné et temporisé qu'il peut êlre paré aux inconvénients de l'interruption de service en séparanl les points endommagés de la distribulion générale.

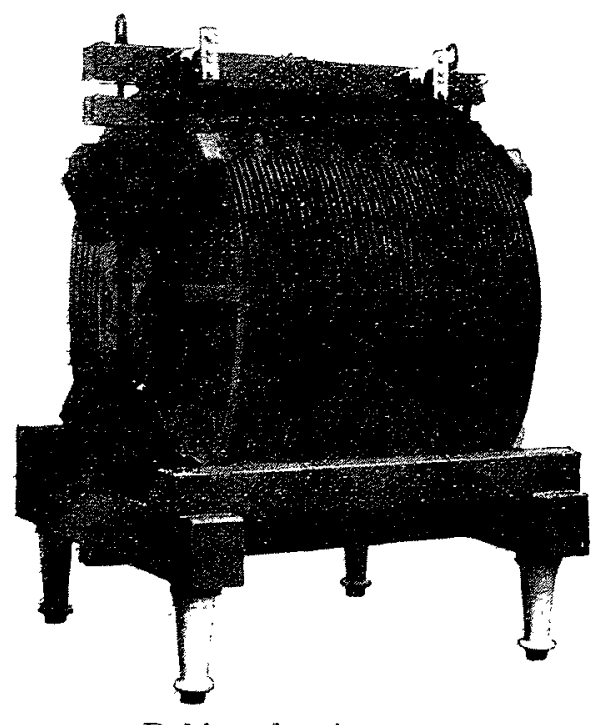

Bobine de réactance.

Pour nous résumer, nous dirons donc qu'à l'heure actuelle, grâce à la pratique acquise à des études minulieuses de condilions du problème et à quelques expérionces particulièrement bien conduites, la construction de l'appareillage d'interruption est sortie de la période de tâtonnements et d'empirisme dans laquelle elle était plongée jusqu’à ces derniers temps.

L'expleitant sait déterminer la puissance maximum interruptible de son réseau, donnée essentielle pour le constructeur gui dimensionne l'appareil suivant des règles facilement applica- bles el contrôlables. Le constructeur étudie, àaprès les lois phy. siques connues, les diffieullés rencontrées.

En parliculier, au cours du développement des tensions do distribution, l'étude des bornes d'amenée et de sortie du courant dans l'interrupleur devint nécessaire. On s'aperçut que les propriétés diélectriques de l'air ne permctaient pas de se contenter de porcelaines d'entréc et de sortie de dimensions plus ou moins grandes à l'intérieur desquelles le conducteur étail engagé axiallement et cntouré d'une couche d'air d'épaisseur variable. L'air, dans ces condilions, est ionnisé par le champ el la traversée ainsi conslituée se perce. Pour éviter cet inconvénient, il faul diminner le champ électrique au voisinge du conducteur. Ceci conduil à des dimensions de traversées prohibitives, de sorle qu'à parlir des tensions de l'ordre de 45.000 volts, on ne construit plus de bornes conlenant de l'air.

A l'heure actuelle, plusieurs dispositions sont connues, et donnent de bous résulats. Dans un premice disposilif, on remplit l'espace annulaire entre le conducteur et la porcelaine soil avec un mélange, huile-résine, soit avec un melange, fluide à chaud, solide à froid, vaseline-résine. Ce disposílif a pour inconvénicnt d'augmenter lo champ dans l'air a la surface extéricure de la traversée, ce qui donne lieu à l'apparition prématurée d'effluves, au moins pour les bornes a très haute tension. Suivant un deuxième disposilif, on constitue la borne par une série de condensateurs en cascade, ce qui a l'avantage d'uniformiser le champ radial tout en donnant une meilleure répartition du champ longiludinal. Les deux dispositions sonl employées concurremment pour les très haules tensions.

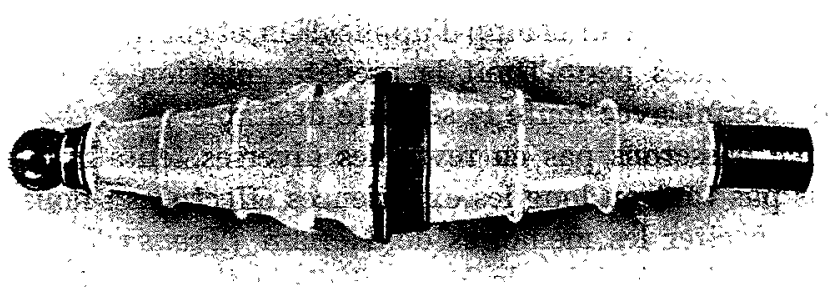

Traverséc sur 70.000 volts.

Ënfiz, on a étudié le champ électritgue en fui-mème, nous nous bornerons à eiter les mémoires de Forstescue (Application of a electrostatic Theorem to a Insulation problem) (Transattions of the A. I. E. E. , Vol. XXX11) de Ch. lice (Experimentat Solution of a Electrostatic Problem) (Transactions of the A. I. E. E., Vol. XXXV1), et de Kuhlmann (Butletin de l'A. S. E. 1916), On a donné aux surfaces des formes convenables pour xéduire les variations trop rapides du champ électrique, ceci afin d'empêcher l'apparition des effluves prématurées, de sorte qu'aujourd'hui la fabrication des bornes est un véritable problème d’électrostatique dont la solution sobtient par des procédés connus en appliquant les admirables travaux de Maxwell et de ses continuateurs.

\section{Etat aCtUel}

Nous avons déerit les progrès réalisés en malière de construction d'appareillage électrique. Voyons quel est son état actuel.

Contrairement à la machine trowrnante, l'interrupteur n'a pas alteint son état limile. En effet, la machîne tournante est douée d'un rendement çu'on nepeut espérer dépasser. On a simplifié et standardisé sa construction et rien ne permel de prévoir que celleci subira un thangement radical; bien plus, tien ne permet de pre vör pourquoi un tel changement serait soubaitable. La machine 
tournante est basée sur les lois de l'induction connues et appliquées depuis longtemps avec une maîtrise absolue. Elle met en jeu les propriétés magnéliques des matériaux industriels, et on a tiré de ceux-ci tout ce qu'ils pouvaicnt raisonnablement donner. Nous pouvons donc dire que lá construction de la machine tournanle, (et aussi du transformateur), semble a l'heure actuolle definitive et immuable, au moins dans ses grandes lignes.

Fin est-il de même de l'interrupteur?

Nous ne lo pensons pas, car si on connaîl en gros les manifeslations de lare de ruplure, son mécanisme inlime nous est inconnu at e'est là pourtant que réside loul le problème de la rupture.

A l'heurc ącluelle, deux conceptions se sont fait jour en malière d'appareillage d'interruption.

La premiere, que nous appellerons " germano-suisse " agit sur l'are un plaçant celui-ci dans un grand volume d'huile et en fracliomant la rüptưré. C’est-à-dire en introduisant le plus grand nombre possible d'arcs en série.

Lidiée fondamentale de celte manière de procéder est celle-ci. Si on dispose plusieurs ares en série, en plaçant convenablement les contacts on effectue plusicurs parcours simultanément, la duré du parcours Lotal des conlacts mobiles se réduit donc à la durée d'un parcours élémentaire. Fin définitive, la vilesse do rupture est augmentée at to temps de rupture diminué.

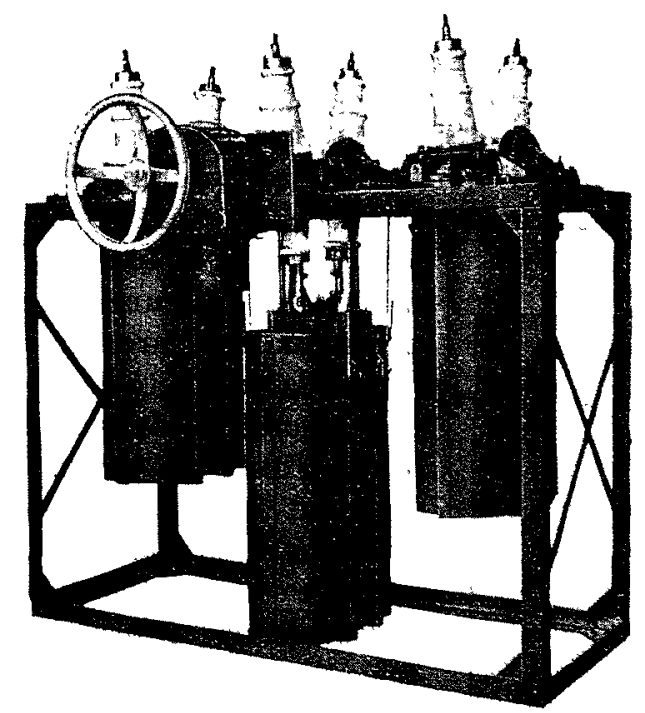

Disioncteur thans l'huile tripolaire 40.000 volts, 250 amp.

Malheureusement, ce raisonnement tombe de lui-mème si on remarque que :

a) Quand on remplace un arc unique de longueur $l$ par $n$ ares égaux en série, la longueur $\lambda$ de chacun de ces arcs est plus grande que $\frac{l}{n}$, l'expérience montre même que $\lambda$ est d'autant plus grand que $\frac{l}{n}$, que $n$ est plus grand ;

b) I a vitesse de rupture dont on parle généralement est la vilesse moyenne. Elle est fonction de la duréce du mouvement. En particulier, si les contacts mobiles sont sollicités seulement par la pesanteur, diminuer l'espace a parcourir $a$ revient aussi entre certaines limiles a diminuer la vitesse, car celle-ci est egale a $\frac{1}{2} \sqrt{2 g \cdot x}$

En définitive, cette solution apparaît comme plutôt illusoire. Elle a en outre l'inconvénient de rendre les appareils encombrants, de conduire à des équipages mobiles plulôt lourds et dont la manotivre exigc parfois des efforts considérablesi
La seconde conception est celle des Anglo-Saxons et principalement des Américains.

Elle consiste à se contenter de deux ruptures en série, (il est plus facile d'en réaliser deux qu'une seule au poinl de vue constructif), mais à prendre toutes les précautions nécessaires pour rendre ces ruptures " effectives " et rapides.

Ceci les a conduit, par exemple, à entourer l'arc d'un cylindre convenablement isolé, dit chambre d'extinction, et dont le rôle consiste à créer une surpression au voisinage de l'arc. Suivant la loi de Paschen, lo potentiel explosif, qui intervient seul à chaque réallumage de l'arc, après le passage du courant par zéro est d'autant plus grand que la pression est plus élevéc. Le produit P.l. de la pression par la distance explosive des électrodes reste au contraire constant. De sorte que si la pression augmente, la longueur d'arc diminue et la rupture ainsi est plus rapide.

Les apparcils ainsi construits conservent tous les avantages d'une extrême simplicité qui peut être jointe à une grande robustesse.

Cependant, malgré los divergences de vues sur certains moyens employés pour augmenter la sécurité du fonctionnement des interrupteurs, il est un certain nombre de points sur lesquels tous les constructeurs, qu'ils procèdent soit de la conception germanosuisse, soit de la conception américaine, sont d'accord. Le plus important est celui de la nécessité d'augmenter les vitesses de rupture. En effet, plus cette vitesse est grande,.plus le temps nécessaire à effectucr la rupture est court, et par conséquent, plus le travail de rupture est diminué. Il résulte de ce qui précède une tendance générale à augmenter la course des contacts mobiles, ce qui présente un double avantage. Celui d'obtenir une plus grande vitesse de séparation de contacts et celui d'avoir dans tous les cas des courses supérieures à la plus grande longueur d'arc possible.

\section{CONCLUSION}

Ceci suffit pour se rendre compte que le dernier mot n'est pas dit en matière d'appareillage d'interruption, Il reste au contraire beaucoup à faire. Il serait extrèmement désirable que des essais systématiques puissent être effectués dans les conditions réelles d'utilisation des appareils. Malheureusement, si le constructeur peut se livrer à toutes sortes d'épreuves électro statiques permettant de vérifier l'isolement, il lui est impossible d'entreprendre les précédentes ou épreuves électro-dynamiques, seules capables de donner une garantie de bon fonctionnement. Le constructeur n'a pas à sa disposition la puissance nécessaire pour faire des essais de rupture en charge. Il serait cependant du plus haut intérêt à la fois du constructeur et de l'exploitant que ces essais puissent être effectués. Il nous semble alors qu'il appartiendrait à un groupement puissant et suffisamment riche, tel que par exemple l'Union des Syndicats d'Electricité, de créer un laboratoire où des ètudes systématiques pourraient être entreprises et dont les exploitants seraient les premiers à profiter.

Nous concluerons en attirant l'attention sur la nécessité d'établir également une réglementation précise en matière de construction et d'essais de réception de l'apparcillage d'interruption pour haute tension, On pourrait reprendre, après études et expériences nouvelles la réglementation du V. D. E. Nerband Deutscher Elektrotechniker) en précisant davantage les conditions de fonctionnement ou "dynamiques " d'emploi des appareils, les règles du V.D. E. concernant en quelque sorte presque exclusivement los conditions d'isolement ou "statiques". Il est de l'intẻrêt de l'exploitant que ne puissent être mis sur le marché des appareils construits en dépit des règles les plus élémentaires de la prudence, si on songo que souvent du bon fonctionnement d'un interrupteur dépend l'avenir' de toute l'installation. 